Article

\title{
Surface Patterns for Drag Modification in Volleyballs
}

\author{
Sungchan Hong ${ }^{1, * \mathbb{C}}$, Takeshi Asai ${ }^{1}$ and Byung Mook Weon ${ }^{2,3, * \mathbb{C}}$ \\ 1 Faculty of Health and Sports Science, University of Tsukuba, Tsukuba-City 305-8574, Japan; \\ asai.takeshi.gf@u.tsukuba.ac.jp \\ 2 Soft Matter Physics Laboratory, School of Advanced Materials Science and Engineering, SKKU Advanced \\ Institute of Nanotechnology (SAINT), Sungkyunkwan University, Suwon 16419, Korea \\ 3 Research Center for Advanced Materials Technology, Sungkyunkwan University, Suwon 16419, Korea \\ * Correspondence: sr7931@hotmail.com (S.H.); bmweon@skku.edu (B.M.W.)
}

Received: 13 August 2019; Accepted: 20 September 2019; Published: 25 September 2019

\begin{abstract}
Surface patterns on objects are important in aerodynamics. We show how surface patterns on volleyballs modify their aerodynamic performances. Conventional volleyballs with six panels show different aerodynamic characteristics along transverse and diagonal directions. Interestingly, isotropic surface patterning with hexagons or dimples enables us to achieve isotropic aerodynamics. This result gives insight into surface-mediated flight controls of projectiles through resisting fluid media.
\end{abstract}

Keywords: aerodynamics; drag crisis; surface pattern; volleyball; wind tunnel experiment

\section{Introduction}

When a spherical ball moves through the air, a long tangle of swirling air trails behind it [1-6]. A turbulent flow causes to slow the ball down. This phenomenon emerges due to drag [4]. At low speeds, the wake is large and the drag is high, while for a ball moving faster than a certain speed, the wake suddenly shrinks and the drag coefficient plummets. The rapid decrease in the drag force is known as drag crisis [4]. The drag crisis occurs when the laminar flow of air in a boundary layer near the ball begins to separate and becomes turbulent $[1,3]$. Competitive players can take advantage of the strange aerodynamic effects in volleyball games [4]. The aerodynamic lift and drag forces can make a volleyball swerve up or down and/or side to side: lift acts in a direction perpendicular to motion and drag acts to directly oppose motion [5]. Drag crisis is still a puzzling phenomenon in fluid mechanics [6].

Ball trajectories are influenced entirely by gravity and aerodynamics [5,7]. The aerodynamic forces are sensitive to the surface properties of balls. Surface modification in balls is therefore an important task: for instance, dimples on golf balls [5]. A moving ball has a high-pressure area on its front side and leaves behind a turbulent wake region, resulting in lower pressure and higher drag. The drag force can be reduced by dimples on a ball when they induce a thin turbulent boundary layer of air on its surface and lower drag because turbulent boundary layer flow has a larger momentum than laminar boundary layer flow and thus delays separation [8]. Dimples allow the smoothly flowing air to follow the ball surface a little farther around the back side and consequently reduce the drag force as the size of the wake decreases. The spinning action of a golf ball makes the air pressure on the bottom of the ball higher than on the top, generating pressure imbalance and an upward lift force on the ball [5]. Since surface roughness plays a significant role in the turbulence dynamics near the surface, there are recent fruitful efforts for flow control through engineering surfaces [9-13]. The aerodynamic drag force on a ball depends on its diameter, speed and surface roughness [14]. The drag force can be smaller on a rough ball than a smooth ball, especially at speeds in ball sports [14]. Zigzag paths (knuckleballs) have been reported for volleyballs with the typical ball velocity and the corresponding 
Reynolds number $\sim 4 \times 10^{5}$ [15]. Practically, aerodynamic modification through pattering surfaces is a plausible task to scientists and engineers [5,16-24]..

In this paper, we demonstrate how surface patterning modifies aerodynamic performance of volleyballs. Particularly we explain why aerodynamic characteristics along transverse and diagonal directions are different in conventional volleyballs with six panels and how isotropic aerodynamics is achieved through isotropic surface patterning with hexagons or dimples. This result gives insight into surface-mediated flight controls of projectiles through resisting fluid media.

\section{Experiments}

As for surface patterns on volleyballs, we selected conventional and surface-modified volleyballs. Conventional volleyballs (Adidas AV514RB and Mizuno 9OV80027) and surface-modified volleyballs (Mikasa MVA200CEV and Molten V5M5000) were purchased commercially. Conventional and surface-modified balls had same diameters of $21 \mathrm{~cm}$, same circumferences of $65 \sim 67 \mathrm{~cm}$ and same weights of $260 \sim 280 \mathrm{~g}$, except for different surface panel numbers and patterns as: six-panel and smooth surface for conventional balls (Adidas AV514RB and Mizuno 9OV80027), while six-panel and hexagon-patterned surface (Molten V5M5000) and eight-panel and dimpled surface (Mikasa MVA200CEV) for surface-modified balls. For the wind tunnel experiments, a circuit flow-type wind tunnel (San Technologies Co., Ltd., Tochigi, Japan) was used at the University of Tsukuba. The maximum wind speed was $55 \mathrm{~m} / \mathrm{s}$, the blow out size was $1.5 \times 1.5 \mathrm{~m}^{2}$ and the wind speed and the turbulence distributions were controlled within $\pm 0.5 \%$ and less than $0.1 \%$, respectively. For the volleyball installed in the wind tunnel, the aerodynamic characteristics were measured with respect to the panel direction. The ball was supported from the back support that was made of a rod with $0.8 \mathrm{~m}$ length and $0.02 \mathrm{~m}$ width. The aerodynamic force of each ball was measured in the range of wind speeds $(U)$ from 7 to $35 \mathrm{~m} / \mathrm{s}$ using a Sting-type 6-division detector (LMC-61256, Nissho Electric Works). The aerodynamic coefficients of the drag coefficient $\left(C_{d}\right)$, the lift force coefficient $\left(C_{l}\right)$ and the side force coefficient $\left(C_{s}\right)$ were calculated as $C_{d}=\frac{2 D}{\rho U^{2} A}, C_{l}=\frac{2 L}{\rho U^{2} A}$ and $C_{s}=\frac{2 S}{\rho U^{2} A}$, respectively. Here $\rho$ is the density of air $\left(\rho=1.2 \mathrm{~kg} / \mathrm{m}^{3}\right), A$ is the cross-sectional area of the volleyball $\left(A=\pi d^{2} / 4 \approx 0.0346 \mathrm{~m}^{2}\right.$ for the diameter of the volleyball $d=0.21 \mathrm{~m}[15]$ ), and the $D, L$, and $S$ quantities are the longitudinal, transverse and side components of the aerodynamic forces [6].

Two intrinsic forces act on a ball moving through the air by competition of gravity and aerodynamics. The first force acts down on the ball, namely, the ball weight $(m g)$, where $m$ is the ball mass and $g=9.80 \mathrm{~m} / \mathrm{s}^{2}$ is the constant magnitude of gravitational acceleration near the Earth surface. The second force is the drag force, whose direction is opposite the ball velocity. By considering that the $x$ axis point along the horizontal and the $y$ axis point vertically upward, the Newton's second law is reduced to $\ddot{x}=-\beta v C_{d} \dot{x}$ and $\ddot{y}=-\beta v C_{d} \dot{y}-g$ where $\beta=\frac{\rho A}{2 m}, v=\sqrt{\dot{x}^{2}+\dot{y}^{2}}$ and the dot signifies the time derivative. These relations can be numerically solved under appropriate initial conditions using a fourth-order Runge-Kutta algorithm. For the speed-dependent $C_{d}$, a linear interpolation between experimental wind-tunnel data points was used. The drag coefficients of each ball taken from the wind tunnel experiments and the corresponding Reynolds numbers were obtained at the same initial speed of $15 \mathrm{~m} / \mathrm{s}$ and the takeoff angle of $25^{\circ}$.

The flight characteristics were investigated by measuring the landing point after being hit. The hitting robot aimed at the center of a non-rotating ball (e.g., float serve) at the typical ball speed of $15 \mathrm{~m} / \mathrm{s}$ in real games $[25,26]$ with a target $\left(1 \times 1 \mathrm{~m}^{2}\right)$ located on the floor $15 \mathrm{~m}$ away. The position at which the volleyball hits the floor was denoted as the landing point with its flight distance along the horizontal and lateral distances. Data analysis was performed from the landing point measurements from the repeated 30 throws for four balls and two panel directions for a total dataset of 240 throws. 


\section{Results and Discussion}

The first question is why aerodynamic characteristics along transverse and diagonal directions are different in conventional volleyballs with six panels. Traditionally, volleyballs are assembled from a total of six panels with each panel formed using three rectangular panel sections for a total of 18 sections and these volleyballs have been used in international competitions over a long period of time, as demonstrated in Figure 1. Conventional volleyballs assembled with six panels inevitably have two different panel directions in the production process. The transverse panel direction is parallel to the panel sections [Figure 1a] and the diagonal panel direction is perpendicular to the point where the three panels meet [Figure 1b]. Assembling volleyballs is like folding a cube with six sides of a square [Figure 1c]. The presence of two panel directions is supposed to be important for the anisotropic aerodynamic performance of volleyballs.

(a)

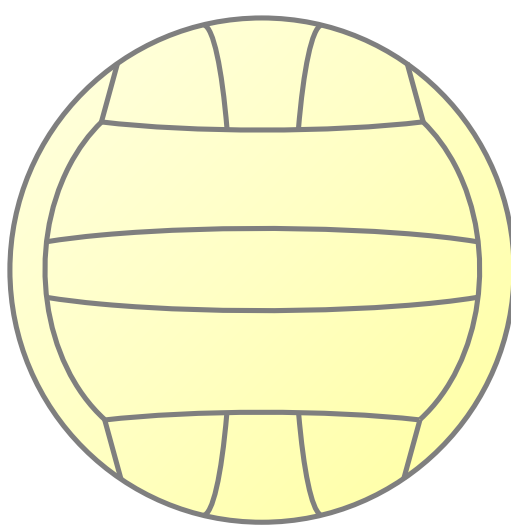

Transverse direction (b)

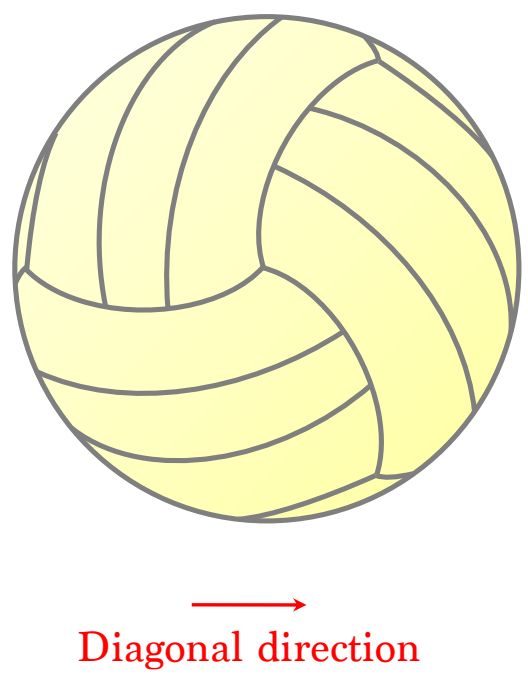

(c)

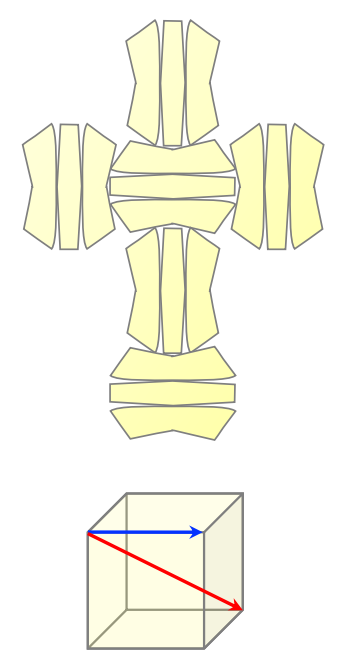

(d)

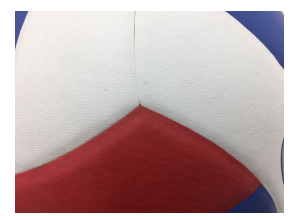

Conv-1

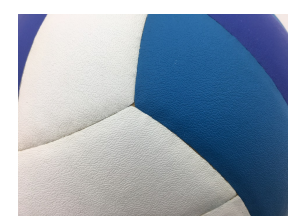

Conv-2

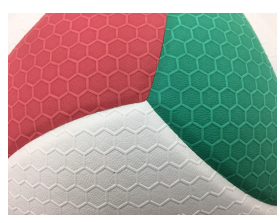

Hexagon

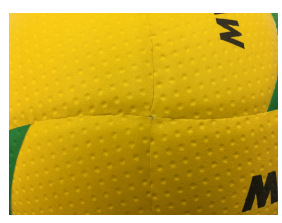

Dimple

Figure 1. Surface patterns of volleyballs with (a) transverse and (b) diagonal directions on panels. (c) In conventional volleyballs, the existence of two panel directions is a result of conventional assembly with six panels. (d) Surface patterns for conventional balls (Conv-1 and Conv-2) and modified balls with hexagon and dimple patterns.

The aerodynamic characteristics of volleyballs are demonstrated in Figure 2. The drag coefficients $C_{d}$ with respect to the Reynolds number $R e$ were measured in the wind tunnel experiments. The $C_{d}-R e$ curves show that the drag crisis is dependent on the panel direction and the surface patterning. The different aerodynamic characteristics along the panel direction are verified for conventional balls: the drag crisis behaves differently between the transverse and the diagonal directions [Figure 3a,b]. The prime cause of the aerodynamic anisotropy is the difference in the panel direction. In contrast, the surface-modified balls have less difference in the drag crisis regardless of the panel direction [Figure 3c,d], which is due to the isotropic surface patterns with hexagons or dimples. 

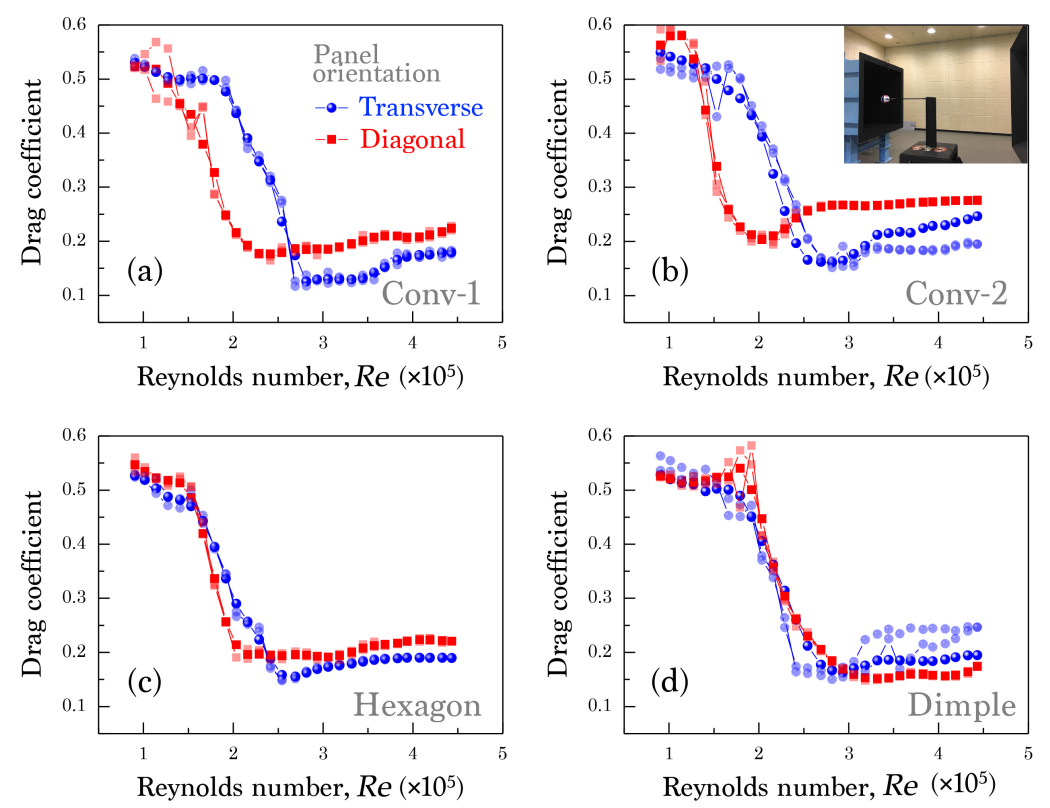

Figure 2. Drag crisis of volleyballs. Circles and squares represent transverse and diagonal directions, respectively, with different surface patterns: (a) Conv-1, (b) Conv-2, (c) Hexagon and (d) Dimple. Inset shows the wind tunnel experiment. To check out the experimental uncertainty, three data were included for each case. Discrepancy in drag crisis appears in conventional balls, while similarity in drag crisis in balls with isotropic patterns.

(a)
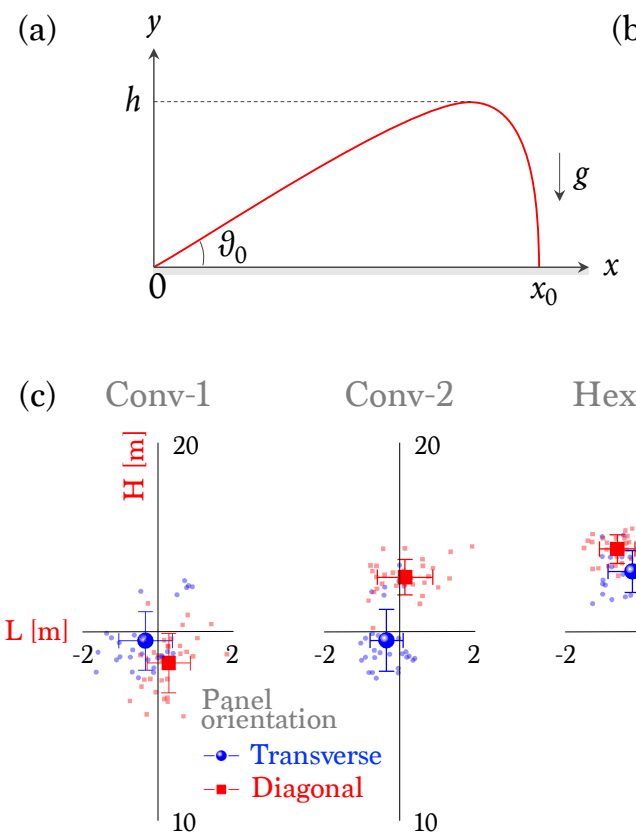

(b)
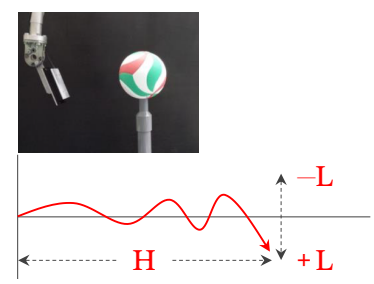

Dimple
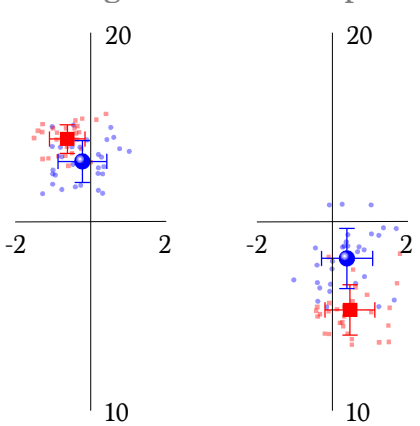

Figure 3. Flight characteristics of volleyballs. (a) An illustration for flight trajectory. (b) Lateral (L) and horizontal $(\mathrm{H})$ flight distances were measured from the landing position experiment using a hitting robot. (c) All data of lateral and horizontal distances are summarized. Each panel with equidistant intervals is depicted at the same range of $\pm 2 \mathrm{~m}$ (lateral) and from 10 to $20 \mathrm{~m}$ (horizontal). Blue and red represents transverse and diagonal directions, respectively. Big circles and squares are the average values with error bars taken from the standard deviations. 
The critical Reynolds numbers $R e_{c}=d U_{c} / v$ for the onset of the drag crisis are measured in Figure 2, where $U_{c}$ is the critical ball velocity and $v$ is the kinematic viscosity of the air $\left(v=1.5 \times 10^{-5} \mathrm{~m} / \mathrm{s}^{2}\right.$ at $\left.20^{\circ} \mathrm{C}[1,15]\right)$.

Figure 3 shows the flight characteristics of each ball taken from the lateral and horizontal landing positions. The longest flight distance (16.6 17.2 $\mathrm{m}$ on average) is found in the Hexagon ball. Interestingly, the shortest flight distance (12.7 14.1 m on average) is observed in the Dimple ball.

The aerodynamic anisotropy of volleyballs originates from the panel direction through the volleyball production process. Importantly, the aerodynamic anisotropy can be eliminated by the isotropic surface modification, for instance, by adopting the isotropic hexagonal patterns and the dimples. For the unmodified (Adidas and Mizuno) volleyballs, the aerodynamics is anisotropic, originating from the panel direction: the drag crisis behaves separately with the transverse and diagonal panel directions. As a result, their flight distances are relatively short. By adopting the surface modification with the isotropic patterns such as hexagonal patterns (Molten) and dimples (Mikasa), the modified volleyballs can have the improved aerodynamics. Their aerodynamic coefficients with respect to the Reynolds number behave almost identically regardless of the panel direction, their aerodynamic forces are stabilized and their flight distances can be controlled to have the longest distance (Molten) or the shortest distance (Mikasa).

The flight distance modification by surface pattern may be explained by the drag coefficients. The Dimple ball, which is currently used in international competitions (e.g., Olympics), has the shorter flight distance compared to the Hexagon ball, which exhibit the longest flight distance. At the ball velocity $U=15 \mathrm{~m} / \mathrm{s}\left(\operatorname{Re} \sim 2 \times 10^{5}\right)$ in the hitting robot tests, the drag coefficients for the Hexagon ball, estimated as $C_{d} \approx 0.26 \sim 0.34$ from Figure 2, are lower than those for the Dimple ball as 0.45 0.50. The conventional balls show the intermediate $C_{d}$ quantities as $0.25 \sim 0.48$ for the Conv- 1 and $0.21 \sim 0.43$ for the Conv-2 balls. Interestingly, the hexagonal pattern in the Hexagon ball significantly reduces the critical Re for the drag crisis, while the dimple pattern in the Dimple ball enhances the critical Re for the drag crisis. Similar results were found in the dimpled soccer balls [27] and the dimpled golf balls [28]. We suppose that the drag enhancement by dimples is associated with the boundary-layer instability.

This study suggests that the critical Re number for the drag crisis can be reduced or enhanced with the surface design in a volleyball. The relatively far or short flight of a ball can be controlled by the surface design of a ball. The lower the drag coefficient, the farther the flight of the ball. The design of the surface pattern can contribute to the drag reduction or the drag enhancement [29]. The surface-modified balls exhibit the quite isotropic aerodynamics and the well-controlled flight distances.

In ball sports, the well-controlled flight trajectory of a ball would be useful to lure opponents and score [15]. For instance, float serves are important skills for ace players and they utilize the knuckle effect of a ball, allowing a ball to fall suddenly or to have irregular flight paths using noor slow-rotating while flying [25]. Therefore, controlling the flight characteristics of a ball would be important to ball designers and players.

This work shows an answer for the hypothesis: from a comprehensive study on the aerodynamic singularity in volleyballs, the present work reveals how the isotropic surface modification can eliminate the aerodynamic anisotropy and reduce or enhance the drag coefficient. As described in Figure 4, the results of lift and side forces show that the isotropy of the aerodynamics would be relevant to the isotropy of the surface pattern. The previous work is limited to identify difference in the critical Reynolds number or the drag coefficient between unmodified and modified volleyballs [19]. Unmodified volleyballs assembled with traditional 18 sections and modified volleyballs with isotropic hexagonal patterns or isotropic dimples represent all feasible designs of volleyballs that are actually used in volleyball games. The main result would be generalizable beyond the specific brands of volleyballs. 

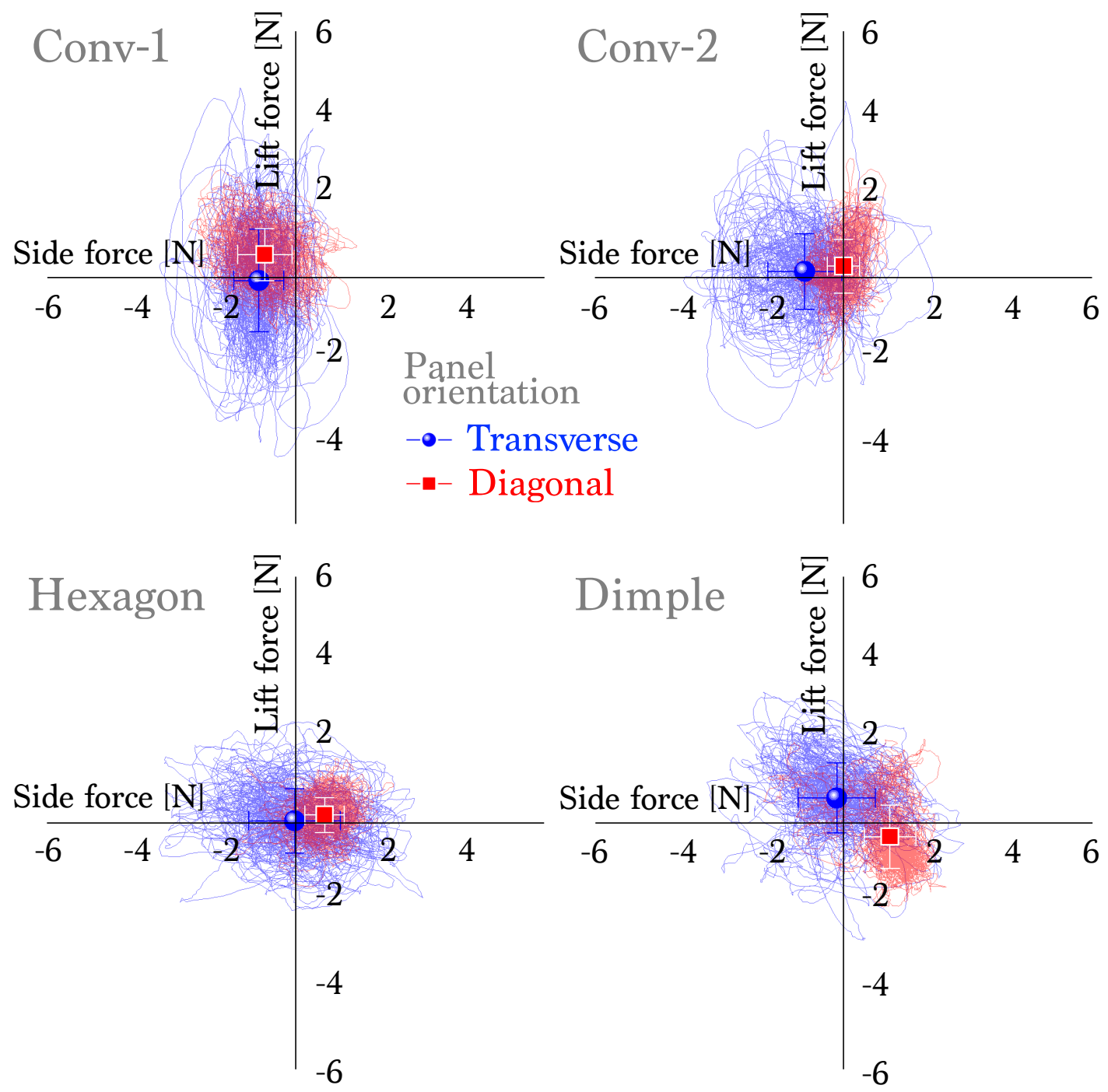

Figure 4. The lift and side forces acting on the ball taken in the wind tunnel experiment $(10 \mathrm{~s}$, wind speed $15 \mathrm{~m} / \mathrm{s}$ ) are quite isotropic from the balls with isotropic (Hexagon and Dimple) surface patterns. Blue and red represents transverse and diagonal directions, respectively. Big circles and squares are the average values with error bars taken from the standard deviations.

Recently, significant changes were tried in design of the volleyball surface. At the 2008 Beijing Olympics, a surface-modified volleyball (Mikasa MVA200) with 8-sheet dimples was introduced as the official ball and in turn adopted in international competitions (e.g., FIVB World League and Olympics). Another volleyball (Molten V5M5000) with hexagonal protrusions on the ball surface was developed and used as the official ball in many league games, including the American League (USA Volleyball) and the National Collegiate Athletic Association (NCAA) League. Similar changes appear in footballs: the aerodynamic force applied to the ball changes significantly depending on the surface panel shapes and consequently affects the aerodynamic performance $[16,17,24,27,30]$. Despite recent developments in design of volleyballs, aerodynamic validation has yet to be reported. For traditional volleyballs, a few reports were found on the numerical simulations [31-33] and the experimental measurements of the flight trajectory or the vortex structure [26,32,34]. For surface-modified volleyballs, a comprehensive understanding of the aerodynamic singularity is still lacking [35]. The surface conditions of volleyballs are different for the tested volleyballs as demonstrated in Figure 2. The aerodynamics and flight characteristics are analyzed from the wind tunnel experiments. 


\section{Conclusions}

To conclude, we demonstrate experimental evidence for the prime cause of the aerodynamic anisotropy in the traditional volleyballs. To eliminate the panel direction dependency, the isotropic surface patterns would be useful. The isotropic surface patterns such as hexagonal protrusions and dimples would significantly contribute to the aerodynamic forces and the final flight distances. This result would be helpful for flight control of projectiles as well as for design of sports balls through resisting media such as air and water, thanks to surface-mediated aerodynamics that would be efficacious by control of drag reduction or enhancement with surface modification.

Author Contributions: Conceptualization, S.H. and B.M.W.; methodology, S.H. and T.A.; software, S.H.; validation, S.H. and B.M.W.; formal analysis, S.H.; investigation, S.H., T.A. and B.M.W.; resources, S.H.; data curation, B.M.W.; writing-original draft preparation, S.H., T.A. and B.M.W.; writing-review and editing, B.M.W.; visualization, S.H. and B.M.W.; supervision, T.A. and B.M.W.; project administration, T.A.; funding acquisition, S.H., T.A. and B.M.W.

Funding: This work was supported by JSPS KAKENHI (Grant No. 15K16442) of the Ministry of Education, Culture, Sports, Science and Technology of the Japanese government and supported by Basic Science Research Program through the National Research Foundation of Korea (NRF) funded by the Ministry of Education (Grant No. 2019R1A6A1A03033215).

Conflicts of Interest: The authors declare no conflict of interest.

\section{References}

1. Frohlich, C. Aerodynamic drag crisis and its possible effect on the flight of baseballs. Am. J. Phys. 1984, 52, 325-334. [CrossRef]

2. Mehta, R.D. Aerodynamics of sports balls. Annu. Rev. Fluid Mech. 1985, 17, 151-189. [CrossRef]

3. Asai, T.; Akatsuka, T.; Haake, S. The physics of football. Phys. World 1998, 11, 5-27. [CrossRef]

4. Cho, A. In volleyball, crafty players serve up an aerodynamic crisis. Science 2004, 306, 42. [CrossRef] [PubMed]

5. Veilleux, T.; Simonds, V. How do dimples in golf balls affect their flight? Sci. Am. 2004, 290, 1.

6. Bot, P.; Rabaud, M.; Thomas, G.; Lombardi, A.; Lebret, C. Sharp transition in the lift force of a fluid flowing past nonsymmetrical obstacles: evidence for a lift crisis in the drag crisis regime. Phys. Rev. Lett. 2016, 117, 234501. [CrossRef] [PubMed]

7. Brancazio, P.J. Equations of sport. Science 1990, 249, 1447-1448. [CrossRef]

8. Choi, J.; Jeon, W.P.; Choi, H. Mechanism of drag reduction by dimples on a sphere. Phys. Fluids 2006, 18, 041702. [CrossRef]

9. Corten, G.P.; Veldkamp, H.F. Damage mitigation techniques in wind turbine blades: A review. Nature 2001, 412, 41-42. [CrossRef]

10. Jiménez, J. Turbulent flow over rough walls. Annu. Rev. Fluid Mech. 2004, 36, 173-196. [CrossRef]

11. Dean, B.; Bhushan, B. Shark-skin surfaces for fluid-drag reduction in turbulent flow: A review. Phil. Trans. R. Soc. A 2010, 368, 4775-4806. [CrossRef] [PubMed]

12. Lauder, G.V.; Wainwright, D.K.; Domel, A.G.; Weaver, J.C.; Wen, L.; Bertoldi, K. Structure, biomimetics, and fluid dynamics of fish skin surfaces. Phys. Rev. Fluids 2016, 1, 060502. [CrossRef]

13. Bocanegra Evans, H.; Hamed, A.M.; Gorumlu, S.; Doosttalab, A.; Aksak, B.; Chamorro, L.P.; Castillo, L. Engineered bio-inspired coating for passive flow control. Proc. Natl. Acad. Sci. USA 2018, 115, 1210-1214. [CrossRef] [PubMed]

14. Cross, R. Effects of turbulence on the drag force on a golf ball. Eur. J. Phys. 2016, 37, 054101. [CrossRef]

15. Texier, B.D.; Cohen, C.; Quere, D.; Clanet, C. Physics of knuckleballs. New J. Phys. 2016, 18, 073027. [CrossRef]

16. Hong, S.; Asai, T. Effect of panel shape of soccer ball on its flight characteristics. Sci. Rep. 2014, 4, 5068. [CrossRef] [PubMed]

17. Hong, S.; Asai, T.; Seo, K. Visualization of air flow around soccer ball using a particle image velocimetry. Sci. Rep. 2015, 5, 15108. [CrossRef] [PubMed]

18. Mehta, R.; Alam, F.; Subic, A. Review of tennis ball aerodynamics. Sports Technol. 2008, 1, 7-16. [CrossRef]

19. Asai, T.; Ito, S.; Seo, K.; Hitotsubashi, A. Aerodynamics of a new volleyball. Procedia Eng. 2010, 2, $2493-2498$. [CrossRef] 
20. Bush, J.W.M. Sports Physics; Clanet, C., Ed.; Les Éditions de l'École Polytechnique: Palaiseau, France, 2013.

21. Cohen, C.; Darbois-Texier, B.; Dupeux, G.; Brunel, E.; Quere, D.; Clanet, C. The aerodynamic wall. Proc. R. Soc. A 2014, 470, 20130497. [CrossRef]

22. Miyazaki, T.; Sakai, W.; Komatsu, T.; Takahashi, N.; Himeno, R. Lift crisis of a spinning table tennis ball. Eur. J. Phys. 2017, 38, 024001. [CrossRef]

23. Cross, R.; Lindsey, C. Measurements of drag and lift on smooth balls in flight. Eur. J. Phys. 2017, $38,044002$. [CrossRef]

24. Goff, J.E.; Kelley, J.; Hobson, C.M.; Seo, K.; Asai, T.; Choppin, S.B. Creating drag and lift curves from soccer trajectories. Eur. J. Phys. 2017, 38, 044003. [CrossRef]

25. Silva, M.; Lacerda, D.; João, D.P.V. Game-related volleyball skills that influence victory. J. Hum. Kinet. 2014, 41, 173-179. [CrossRef] [PubMed]

26. MacKenzie, S.; Kortegaard, K.; LeVangie, M.; Barro, B. Evaluation of two methods of the jump float serve in volleyball. J. Appl. Biomech. 2012, 28, 579-586. [CrossRef] [PubMed]

27. Hong, S.; Asai, T. Aerodynamic effects of dimples on soccer ball surfaces. Heliyon 2017, 3, e00432. [CrossRef]

28. Chowdhury, H.; Loganathan, B.; Wang, Y.; Mustary, I.; Alam, F. A study of dimple characteristics on golf ball drag. Procedia Eng. 2016, 147, 87-91. [CrossRef]

29. Rastegari, A.; Akhavan, R. The common mechanism of turbulent skin-friction drag reduction with superhydrophobic longitudinal microgrooves and riblets. J. Fluid Mech. 2018, 838, 68-104. [CrossRef]

30. Oggiano, L.; Sætran, L. Aerodynamics of modern soccer balls. Procedia Eng. 2010, 2, 2473-2479. [CrossRef]

31. Coleman, S.G.; Benham, A.S.; Northcott, S.R. A three-dimensional cinematographical analysis of the volleyball spike. J. Sports Sci. 1993, 11, 295-302. [CrossRef]

32. Jalilian, P.; Kreun, P.K.; Makhmalbaf, M.M.; Liou, W.W. Computational aerodynamics of baseball, soccer ball and volleyball. Am. J. Sports Sci. 2014, 2, 115-121. [CrossRef]

33. Kao, S.S.; Sellens, R.W.; Stevenson, J.M. A mathematical model for the trajectory of a spiked volleyball and its coaching application. J. Appl. Biomech. 1994, 10, 95-109. [CrossRef]

34. Wei, Q.; Lin, R.; Liu, Z. Vortex-induced dynamic loads on a non-spinning volleyball. Fluid Dyn. Res. 1988, 3 , 231-237.

35. Asai, T.; Ito, S.; Seo, K.; Hitotsubashi, A. Fundamental aerodynamics of a new volleyball. Sports Technol. 2010, 3, 235-239. [CrossRef]

(C) 2019 by the authors. Licensee MDPI, Basel, Switzerland. This article is an open access article distributed under the terms and conditions of the Creative Commons Attribution (CC BY) license (http://creativecommons.org/licenses/by/4.0/). 